ARTICLE

Received 9 Dec 2014 | Accepted 9 Mar 2015 | Published 12 May 2015

\title{
Anammox Planctomycetes have a peptidoglycan cell wall
}

\author{
Muriel C.F. van Teeseling ${ }^{1}$, Rob J. Mesman¹, Erkin Kuru², Akbar Espaillat ${ }^{3}$, Felipe Cava ${ }^{3}$, Yves V. Brun ${ }^{4}$, \\ Michael S. VanNieuwenhze ${ }^{5}$, Boran Kartal ${ }^{1,6}$ \& Laura van Niftrik ${ }^{1}$
}

Planctomycetes are intriguing microorganisms that apparently lack peptidoglycan, a structure that controls the shape and integrity of almost all bacterial cells. Therefore, the planctomycetal cell envelope is considered exceptional and their cell plan uniquely compartmentalized. Anaerobic ammonium-oxidizing (anammox) Planctomycetes play a key role in the global nitrogen cycle by releasing fixed nitrogen back to the atmosphere as $\mathrm{N}_{2}$. Here using a complementary array of state-of-the-art techniques including continuous culturing, cryotransmission electron microscopy, peptidoglycan-specific probes and muropeptide analysis, we show that the anammox bacterium Kuenenia stuttgartiensis contains peptidoglycan. On the basis of the thickness, composition and location of peptidoglycan in K. stuttgartiensis, we propose to redefine Planctomycetes as Gram-negative bacteria. Our results demonstrate that Planctomycetes are not an exception to the universal presence of peptidoglycan in bacteria.

\footnotetext{
${ }^{1}$ Department of Microbiology, Institute for Water and Wetland Research, Faculty of Science, Radboud University, Nijmegen 6525AJ, The Netherlands.

2 Interdisciplinary Biochemistry Program, Indiana University, Bloomington, Indiana 47405, USA. ${ }^{3}$ Department of Molecular Biology and Laboratory for Molecular Infection Medicine Sweden, Umeå Centre for Microbial Research, Umeå University, Umeå SE-90187, Sweden. ${ }^{4}$ Department of Biology, Indiana University, Bloomington, Indiana 47405, USA. ${ }^{5}$ Department of Chemistry, Indiana University, Bloomington, Indiana 47405, USA. ${ }^{6}$ Department of Biochemistry and Microbiology, Laboratory of Microbiology, Gent University, Gent 9000, Belgium. Correspondence and requests for materials should be addressed to B.K. (email: kartal@science.ru.nl).
} 
$\mathrm{M}$ aintaining cellular integrity is crucial for life and in particular challenging for small, unicellular organisms. In bacteria, the virtually universal solution to this problem is the presence of peptidoglycan, which also determines the cell shape and facilitates cell growth and division ${ }^{1}$. Peptidoglycan is a mesh-like heteropolymer consisting of a lysozyme-sensitive sugar backbone of alternating $\mathrm{N}$-acetylglucosamine (GlcNAc) and $N$-acetylmuramic acid (MurNAc) residues cross-linked by short D-amino acid-rich peptide stems attached to each of the MurNAc residues ${ }^{1}$. Typically, the nascent peptide stem is a pentapeptide with the sequence of one L-alanine, one D-glutamate, one diamino acid and two D-alanines.

Traditionally, bacteria are classified into two groups based on their cell envelope properties. In Gram-positive bacteria, the peptidoglycan layer is relatively thick (15-30 nm), typically contains L-lysine (L-Lys) as the third amino acid and is located outside the cytoplasmic membrane, which is the outermost membrane of the cell ${ }^{1}$. The Gram-negative peptidoglycan is relatively thin (1.5$15 \mathrm{~nm}$ ), typically contains meso-diaminopimelic acid (meso-DAP) instead of L-Lys and is located between the cytoplasmic and the Gram-negative specific outer membrane ${ }^{1}$.

Planctomycetes are extraordinary organisms that belong to the evolutionarily deep-branching bacterial superphylum of Planctomycetes, Verrucomicrobia and Chlamydiae ${ }^{2}$. Both Planctomycetes $^{3-5}$ and Chlamydiae ${ }^{6}$ have been proposed to be among the few exceptions lacking peptidoglycan, which is one of the most conserved structural characteristics of bacteria. However, chlamydial species are commonly sensitive to antibiotics targeting peptidoglycan and have most of the genes involved in its biosynthesis, and the presence of peptidoglycan has recently been shown in some chlamydial species ${ }^{7,8}$. Paradoxically, although the free-living Planctomycetes are expected to need a peptidoglycan shell more than the intracellular and therefore osmotically protected Chlamydiae, they are usually insensitive to antibiotics targeting peptidoglycan ${ }^{3,5}$, are reported to lack a varying number of genes crucial for its biosynthesis 9,10 , and biochemical analyses failed to show the peptidoglycan components meso-DAP and MurNAc in isolated cell envelopes of all eight previously tested Planctomycete strains $^{3}$.

Most planctomycete species, like typical Gram-negative bacteria, have two compartments enclosed by membranes. In Planctomycetes, the innermost membrane is unusually curved ${ }^{11}$. Historically, the planctomycetal outermost membrane was defined as a cytoplasmic membrane $e^{11,12}$. However, based on the bioinformatic analysis (focusing on marker genes for outer membrane protein and lipopolysaccharide insertion ${ }^{13}$ ), the outermost membrane has recently been proposed to be an outer membrane typical of Gram-negative bacteria ${ }^{13-15}$. On the basis of these hypotheses, Planctomycetes can be defined as either uniquely compartmentalized or Gram-negative bacteria. In the absence of any apparent peptidoglycan, arguments for the first hypothesis found considerable support ${ }^{18-18}$ and a shared evolutionary link between Planctomycetes and Eukaryotic cells was suggested ${ }^{16-19}$. Therefore, we argue that showing the presence of peptidoglycan in Planctomycetes and elucidating its characteristics would resolve the controversy concerning the Planctomycete-specific cell envelope and cell plan ${ }^{11-15,20}$ and its contentious link to the origins of eukaryotic cells ${ }^{16-19}$.

Anaerobic ammonium-oxidizing (anammox) bacteria form a distinct, phylogenetically deep-branching group within the Planctomycetes 9 . These microorganisms convert ammonium and nitrite to dinitrogen gas via nitric oxide and hydrazine as intermediates ${ }^{21}$. They occur in aquatic and terrestrial environments and play a crucial role in the biological nitrogen cycle estimated to produce approximately half of the dinitrogen gas present in the atmosphere ${ }^{22}$. Furthermore, the anammox process is widely applied to remove ammonium from wastewater $^{23}$.

Compared with most other Planctomycetes, the anammox cell contains an additional, third, membrane-enclosed compartment ${ }^{11,24}$. This innermost compartment, the anammoxosome, harbours the catabolic machinery and is surrounded by the cytoplasm (also known as riboplasm), which contains the ribosomes and nucleoid ${ }^{24,25}$. Depending on the interpretation of the S-layer-enclosed outermost membrane ${ }^{26}$, the outermost compartment is either a unique cytoplasmic compartment called the paryphoplasm (in accordance with the historical definition) or a periplasm typical for a Gram-negative cell envelope. Unlike other Planctomycetes that divide by budding9,16, anammox bacteria divide by binary fission without the canonical cell division ring protein $\mathrm{FtsZ}^{27}$

Similar to other Planctomycetes, the cell envelope of anammox bacteria was proposed to lack peptidoglycan. Since no anammox bacteria were included in the initial peptidoglycan-targeting biochemical analyses ${ }^{3}$, the absence of peptidoglycan in anammox bacteria was based on the absence of a peptidoglycan layer in the outermost anammox compartment of resin-embedded sections of either high-pressure frozen and freeze-substituted or chemically fixed anammox cells ${ }^{11,28}$. However, all genes essential for peptidoglycan biosynthesis are present in the genome of the anammox bacterium Kuenenia stuttgartiensis, apart from homologs of peptidoglycan-specific glycosyltransferase (GT) class 51 (CAZy database ${ }^{29}$ ) necessary to polymerize the sugar backbone. Despite the apparent lack of these GTs, it was recently shown $^{30}$ that $K$. stuttgartiensis cells lyse with lysozyme in the presence of EDTA and their growth is inhibited by penicillin G.

Here we redefine the anammox Planctomycete K. stuttgartiensis as a Gram-negative bacterium based on the discovery and characterization of a peptidoglycan layer contained within the cell envelope. To this end, we use a complementary array of stateof-the-art techniques including continuous culturing, cryo-transmission electron microscopy (cryoTEM), incorporation of peptidoglycan-specific probes imaged with structured illumination microscopy (SIM) and ultrasensitive ultra performance liquid chromatography (UPLC)-based muropeptide analysis.

\section{Results}

CryoTEM shows hitherto unobserved cell envelope layer. To study the cell envelope of $K$. stuttgartiensis, we used a highly enriched $(>95 \%)$ culture of free-living, planktonic cells. Cryoelectron microscopy of vitreous sections ${ }^{31}$, reflecting the nearnative hydrated state of the cells, showed a layer in the cell envelope that was previously unobserved. This electron-dense layer was located underneath the outermost membrane (Fig. 1). The thickness of the layer varied between 4.5 and $6 \mathrm{~nm}$, which is in the range reported for peptidoglycan in Gram-negative bacteria $^{1}$. The location of this layer, in the outermost cell compartment, matched the location of peptidoglycan in Gramnegative bacteria and therefore this layer was a good candidate to represent the peptidoglycan shell in this bacterium.

Peptidoglycan isolation yields lysozyme-sensitive sacculi. After they were harvested from an enrichment culture (>95\%), $K$. stuttgartiensis cells were further enriched to $99.9 \%$ purity using density centrifugation. When this highly enriched sample was boiled in SDS and negatively stained with uranyl acetate, cellshaped structures similar to peptidoglycan sacculi were recovered (Fig. 2a, Supplementary Fig. 1). In rare instances, sacculi with a figure-eight shape, probably obtained from dividing K. stuttgartiensis cells, were also detected (Supplementary Fig. 2). 

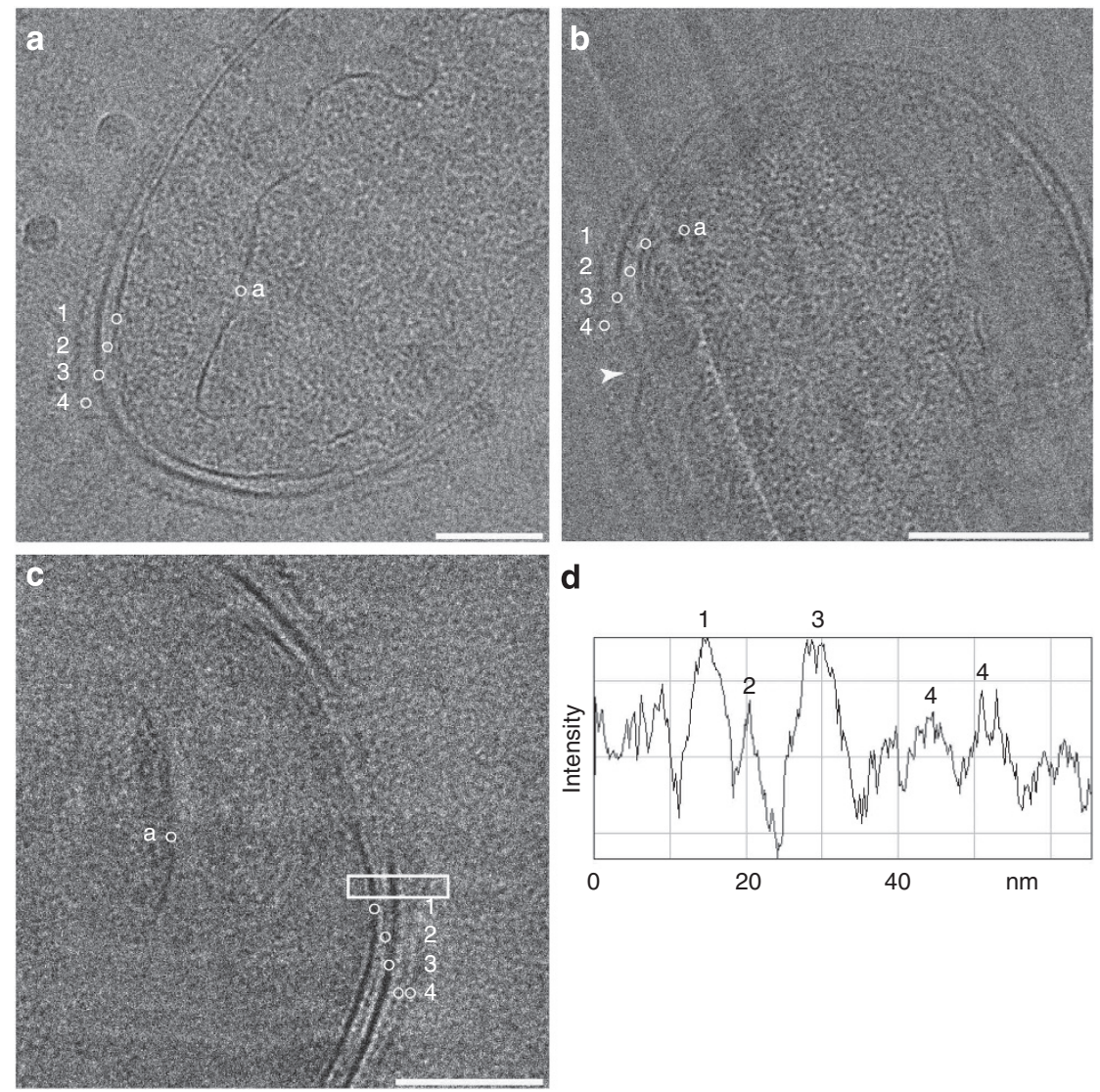

Figure 1 | CryoTEM of vitreous sections revealed a previously unobserved layer in the cell envelope of $\boldsymbol{K}$. stuttgartiensis. (a-c) Single $K$. stuttgartiensis cells as observed with cryo-electron microscopy of vitreous sections, in which all membranes and cell envelope layers have been annotated. (b) A dividing K. stuttgartiensis cell, the division site is indicated with an arrowhead. (d) The intensity profile of the area in the box in (c) encompassing the cell envelope verified the extra layer to be a separate entity. a, anammoxosome membrane; 1, cytoplasmic membrane; 2, putative peptidoglycan; 3, outer membrane; 4 , S-layer. Scale bars, $100 \mathrm{~nm}$.
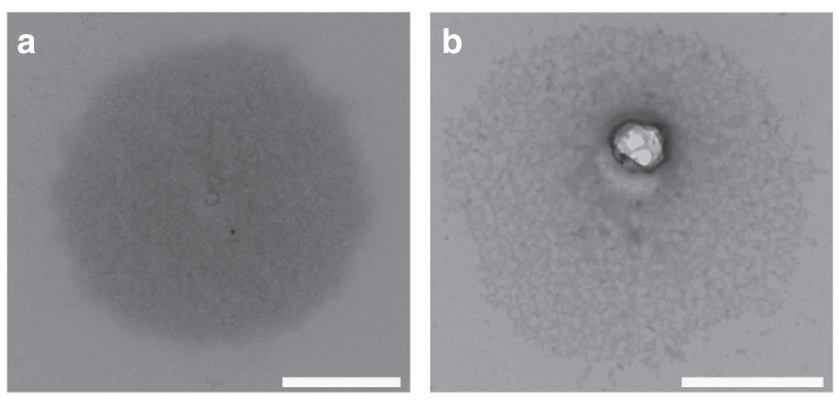

Figure 2 | Lysozyme-sensitive sacculi were obtained by boiling $K$. stuttgartiensis cells enriched by density centrifugation in SDS. (a) TEM of K. stuttgartiensis sacculus using negative staining. (b) After lysozyme treatment, the $K$. stuttgartiensis sacculi were absent or had a fibrous appearance, as observed by negative staining via TEM. Scale bars, $1 \mu \mathrm{m}$.

When $K$. stuttgartiensis sacculi were incubated for $5 \mathrm{~h}$ with lysozyme, which cleaves peptidoglycan by hydrolysing the $\beta-1,4$ bonds between the two sugar components MurNAc and $\mathrm{GlcNAc}^{32}$, the sacculi disintegrated and only fibrous material was visible (Fig. 2b). This was consistent with the previous observation of fibrous material upon enzymatically cleaving the sugar backbone of Escherichia coli peptidoglycan sacculi ${ }^{33}$. The disintegration of the sacculi was solely dependent on the addition of lysozyme, as sacculi incubated without lysozyme remained intact. This suggested that the sacculi were indeed composed of a peptidoglycan-like sugar backbone. Interestingly, this observation also indicated that $K$. stuttgartiensis must be encoding and expressing at least one polymerizing GT class 51 enzyme or another unknown enzyme that polymerizes the peptidoglycan sugar backbone. The apparent absence of such a gene from the $K$. stuttgartiensis genome could be due to the fact that the genome is currently only $\geq 98 \%$ complete $^{34}$. However, according to the CAZy database ${ }^{29}$ other Planctomycetes, as well as Chlamydiae ${ }^{6}$, also lack GT class 51 enzymes and therefore it seems more likely that the enzyme is a member of a new, perhaps Planctomycetesand Chlamydia-specific, uncharacterized class of GTs.

Peptidoglycan peptide stem was verified by specific probes. The peptidoglycan biosynthesis machinery is promiscuous ${ }^{35}$ and therefore non-canonical D-amino acid compounds, such as fluorescent or bio-orthogonal D-amino acids or bio-orthogonal D-amino acid containing dipeptides, can be incorporated at the C-terminus of the peptide stems. This has recently been exploited to specifically stain peptidoglycan ${ }^{8,36,37}$. When grown for a part of the cell division cycle $(10-14 \%)$ in the presence of the bioorthogonal D-amino acid dipeptide ethynyl-D-alanyl-D-alanine (EDA-DA), K. stuttgartiensis cells displayed the characteristic fluorescent signal of probe incorporation after coupling with a fluorophore (Fig. 3b,c). Comparable to peptidoglycan labelling observed in other microorganisms ${ }^{36}$, most of the D-amino acid dipeptide incorporation occurred at the cell division site as 

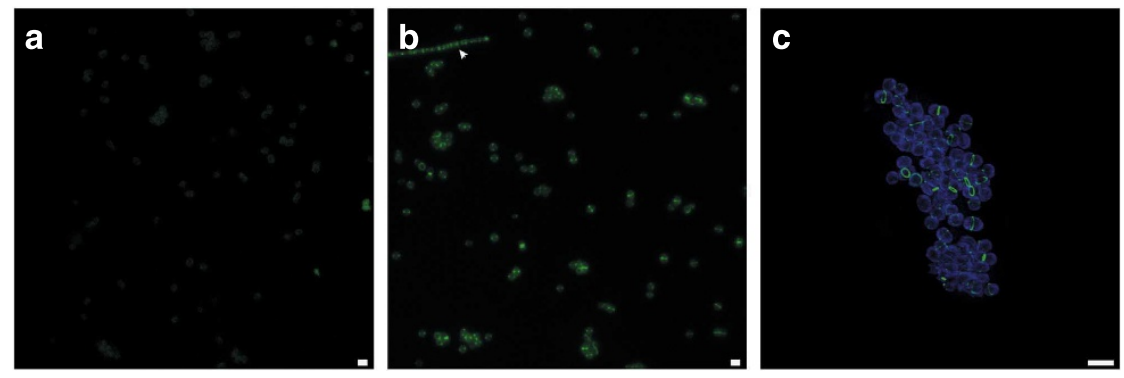

Figure 3 | Fluorescence microscopy of $K$. stuttgartiensis cells grown in the presence of peptidoglycan-specific $D$-alanine dipeptide probes indicated the presence of peptidoglycan. (a) The negative control probe ELA-LA, which cannot be incorporated, shows only a faint background. (b) Septal incorporation of EDA-DA is present both in anammox and rod-shaped non-anammox species present in the bioreactor (arrowhead). (c) SIM clearly shows that EDA-DA was incorporated specifically at the cell division site. Probe incorporation was visualized with a complimentary fluorophore using click chemistry (green) and cell surfaces were labelled by amine-reactive Pacific Blue N-hydroxysuccinimide ester (NHS) (blue). ELA-LA, ethynyl-L-alanyl-L-alanine. Scale bars, $2 \mu \mathrm{m}$.

determined by fluorescence and super-resolution SIM, while experiments with L-amino-acid dipeptide controls only resulted in background fluorescence (Fig. 3a).

Muropeptide analysis confirms sacculi contain peptidoglycan. The structure of the peptidoglycan subunits (muropeptides) was determined by UPLC and subsequent mass spectrometry (Fig. 4a). Muropeptides were obtained by muramidase degradation of sacculi prepared from Histodenz-purified K. stuttgartiensis cells (99.9\%). Indeed, GlcNAc-MurNAc coupled to 2-4 amino acids (M2-M4) as well as cross-linked fragments (D43 and presumably D44) were detected in $K$. stuttgartiensis sacculi (Fig. 4b,c). The largest monomeric peptidoglycan fragment obtained (M4) was identified as GlcNac-MurNAc-L-Ala-D-Glumeso-DAP-D-Ala. Strikingly, the third amino acid was identified as meso-DAP (Fig. 4), which is typical for Gram-negative peptidoglycan ${ }^{1}$. In agreement with this observation, all genes necessary for meso-DAP synthesis are present in the $K$. stuttgartiensis genome (Supplementary Table 1). Furthermore, the predicted protein sequence for MurE, which couples the third amino acid to the peptidoglycan precursors, has the arginine residue (Arg416 in E. coli) necessary for specifically incorporating meso-DAP (instead of L-Lys) into the peptidoglycan precursor ${ }^{38}$.

Peptidoglycan from $K$. stuttgartiensis had relatively little M4 and D44 compared with E. coli K12. In K. stuttgartiensis, M3 was the most abundant monomer, which suggested that the enzyme cleaving between the third (meso-DAP) and fourth (D-Ala) amino acid in the peptide stem (L,D-endopeptidase) was highly active. Further investigation will be necessary to determine whether the abundance of M3 over M4 has an impact on the biology of the bacterium. The presence of D43 cross-linked muropeptides indicated the expression of D,D-transpeptidases, which was supported by the identification of homologs to $\mathrm{Pbp} 2$ and $\mathrm{Pbp} 3$ encoded in the genome of $K$. stuttgartiensis (Supplementary Table 1).

\section{Discussion}

This study presents the first experimental evidence of peptidoglycan in an anammox Planctomycete. We show that K. stuttgartiensis has a relatively thin, meso-DAP-containing peptidoglycan layer located underneath its outer membrane, which fits to the typical characteristics of Gram-negative peptidoglycan. The location of the peptidoglycan in anammox bacteria is striking since the non-FtsZ containing cell division ring $^{27}$ was observed in the exact same compartment of the cell. To the best of our knowledge, a cell division ring has not been previously observed in the peptidoglycan-containing compartment of any bacterium and this further substantiates that anammox bacteria divide via a unique mechanism. On the basis of our investigations, facilitated by new methodology that recently became available, and the results of Jeske et al. ${ }^{39}$ that present similar findings in other planctomycetal lineages, we propose to redefine the Planctomycetes as Gram-negative microorganisms and therefore end the longstanding controversy about the planctomycetal cell plan. Taken together, these findings clearly show that Planctomycetes are not an exception to the universal rule of peptidoglycan cell walls in bacteria, and consequently all free-living bacteria possess peptidoglycan. These findings also lead us to conclude that an evolutionary link between Planctomycetes and eukaryotes is unlikely.

\section{Methods}

K. stuttgartiensis enrichment culture. Free-living planktonic K. stuttgartiensis cells were grown in an enrichment culture ( $\sim 95 \% \mathrm{~K}$. stuttgartiensis) in a membrane bioreactor as described previously ${ }^{40}$. In short, the reactor (working volume 11) was fed continuously with mineral medium ${ }^{41}$ containing $45 \mathrm{mM}$ nitrite and ammonium (each) at a flow rate of $4.2 \mathrm{ml} \mathrm{min}^{-1}$ ( $\sim 61$ per day). The $\mathrm{pH}$ of the reactor was controlled at 7.3 with a potassium bicarbonate solution $\left(100 \mathrm{gl}^{-1}\right)$. The reactor was operated at $33^{\circ} \mathrm{C}$ and was stirred at 300 r.p.m. A gas mixture of $\mathrm{Ar} / \mathrm{CO}_{2}(95 / 5 \%)$ with a flow of $10 \mathrm{ml} \mathrm{min}{ }^{-1}$ was supplied to the reactor to maintain anaerobic conditions. The cells were washed out the reactor continuously (1.11 per day) to maintain the cell density of the culture (optical density at $600 \mathrm{~nm}$ was $1.1-1.2)$.

Cryo-transmission electron microscopy. K. stuttgartiensis cells $(4 \mathrm{ml})$ were pelleted $\left(4 \mathrm{~min}, 600 \mathrm{~g}, 33^{\circ} \mathrm{C}\right)$ and resuspended in a small amount $(50 \mu \mathrm{l})$ of mineral medium containing $1.25 \mathrm{gl}^{-1}$ sodium bicarbonate and trace minerals ${ }^{41}$ and left to recover for $15 \mathrm{~min}$ at $33^{\circ} \mathrm{C}$. Cells were gently mixed with an equal volume of $40 \%$ dextran $\left(70 \mathrm{kD}\right.$ molecular weight) in mineral medium ${ }^{41}$, loaded in copper tubes and high-pressure frozen (HPM100 (Leica Microsystems, Vienna, Austria)). For preparing vitreous sections, copper tubes were loaded in a cryo-ultramicrotome (Leica FC7/UC7, Leica Microsystems) pre-cooled at -145 to $-150^{\circ} \mathrm{C}$. Tubes were trimmed to a pyramid with a 50 by $50 \mu \mathrm{m}$ block face using a cryo-trim $20^{\circ}$ diamond knife (Diatome, Biel, Switzerland). Frozen-hydrated sections (45 nm) for cryo-electron microscopy of vitreous sections were sectioned on a $30^{\circ}$ cryoimmuno diamond knife (Diatome). Sections were attached to carbon-coated 200 mesh copper grids (Stork-Veco, Eerbeek, Netherlands) by electrostatic force using the Crion system (Leica Microsystems). Grids were transferred to the loading station of a high-tilt cryotomography holder (914 (Gatan, Munich, Germany)) under liquid nitrogen. Sections were imaged in a JEOL (Tokyo, Japan) JEM 2100 Transmission Electron Microscope operated at $200 \mathrm{kV}$ in low-dose mode. Images were recorded using the Gatan F4000 bottom-mount camera.

Isolation of peptidoglycan sacculi. K. stuttgartiensis cells were further enriched from the other microorganisms in the enrichment culture by density centrifugation $(15 \mathrm{~min}, 1,000 \mathrm{~g})$ on a $15-30 \%$ Histodenz gradient in $20 \mathrm{mM}$ HEPES buffer $\mathrm{pH} 7.5$ including $1 \mathrm{gl}^{-1}$ sodium bicarbonate. A distinct red band indicating the presence of $K$. stuttgartiensis was present approximately halfway through the gradient. Here $K$. stuttgartiensis presence and abundance was verified by fluorescence in situ hybridization microscopy using the specific Amx820 (ref. 42) and Pla46 (ref. 43) probes. Cells were boiled in $4 \%$ SDS for $60 \mathrm{~min}$ at $100^{\circ} \mathrm{C}$ and applied to a glow- 


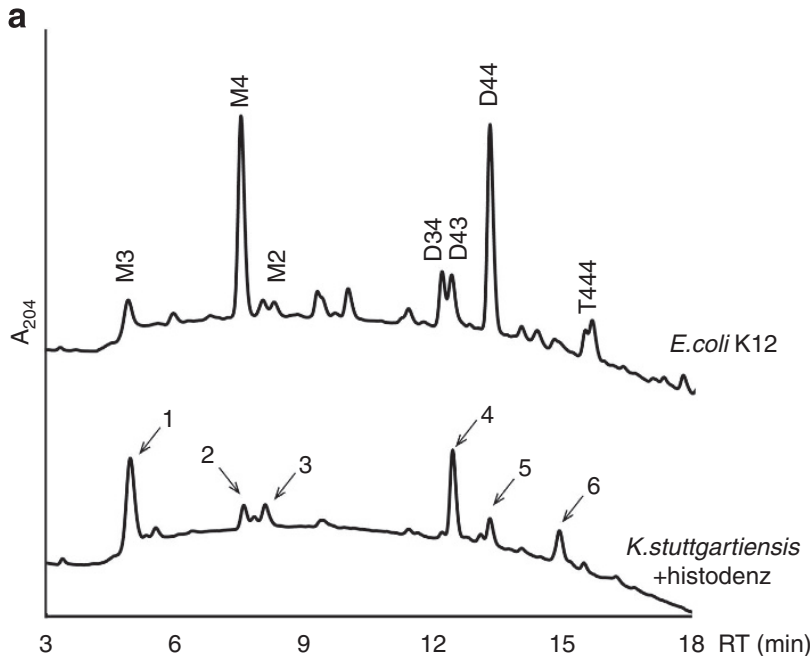

b

\begin{tabular}{ccccccc} 
Peak & RT & $\%$ & {$[\mathrm{M}+\mathrm{H}]^{+}$theo } & {$[\mathrm{M}+\mathrm{H}]^{+}$det } & $\begin{array}{c}\text { Difference } \\
\text { (theo-det) }\end{array}$ & Muropeptide \\
\hline 1 & 5 & 31.5 & 871.38 & 871.37 & 0.01 & $\mathrm{M} 3$ \\
2 & 7.6 & 5.8 & 942.41 & 942.36 & 0.05 & $\mathrm{M} 4$ \\
3 & 8.1 & 6.9 & 699.29 & 699.27 & 0.02 & $\mathrm{M} 2$ \\
4 & 12.5 & 22.3 & 1794.77 & 1794.70 & 0.07 & $\mathrm{D} 43$ \\
5 & 13.3 & 5.7 & 1865.81 & ND & - & $\mathrm{D}^{*}$ \\
6 & 15 & 10.1 & - & $\mathrm{ND}$ & - & - \\
\hline
\end{tabular}

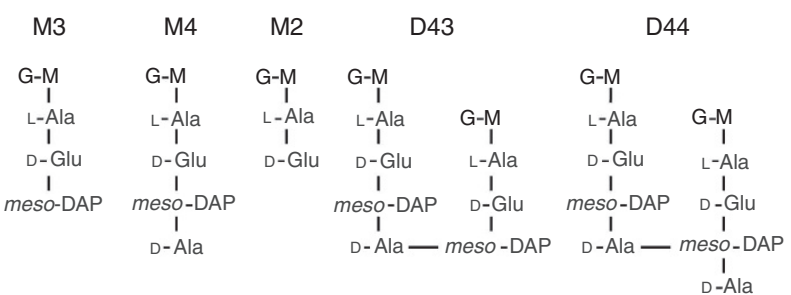

Pd catalyst ( 0.2 p.p.m. residual $\mathrm{O}_{2}$ ). The cells were supplied with the abovementioned HEPES buffer containing $7 \mathrm{mM} \mathrm{NH}_{4}^{+}$and $\mathrm{NO}_{2}^{-}$each and the peptidoglycan-specific probe EDA-DA $(1 \mathrm{mM})$ with a flow rate of $5 \mathrm{ml} \mathrm{h}^{-1}$ for $12-15 \mathrm{~h}$. The fed-batch reactors were stirred at 150 r.p.m. and incubated in the dark at $30^{\circ} \mathrm{C}$. As a control, cells were grown under the same conditions in the presence of ethynyl-L-alanine-L-alanine (ELA-LA) $(1 \mathrm{mM})$. Cells were harvested, washed three times in the above-mentioned HEPES buffer $(3,000 \mathrm{~g}, 10 \mathrm{~min})$ and resuspended in $4 \mathrm{ml}$ HEPES buffer. The resuspended cells were put in an equal volume of $4 \%$ paraformaldehyde in the HEPES buffer and incubated for $20 \mathrm{~min}$ at room temperature and subsequently for $90 \mathrm{~min}$ at $4{ }^{\circ} \mathrm{C}$. The fixed cells were pelleted by centrifugation $(11,700 \mathrm{~g}, 5 \mathrm{~min})$ and resuspended in $0.5 \mathrm{ml} 0.1 \%$ paraformaldehyde for temporary storage. Pelleted cells grown in the presence of EDA-DA and ELA-LA. were permeabilized by incubating them for $5 \mathrm{~min}$ in $1.5 \mathrm{ml}$ PBS with $0.25 \%$ Triton X-100. The cells were washed once in PBS, and the fluorophore Alexa 488 -azide $(20 \mu \mathrm{M})$ was attached via a copper(I) catalysed click reaction (incubation $60 \mathrm{~min}$ in $1.5 \mathrm{ml}$ at room temperature in the Click-iT Cell Reaction Buffer Kit (ThermoFischer, Waltham, USA). Afterwards, cells were washed three times in PBS $(1.5 \mathrm{ml})$, incubated $20 \mathrm{~min}$ at room temperature in the presence of $5 \mu \mathrm{gl}^{-1}$ Pacific Blue NHS ester (ThermoFischer, Waltham, USA), washed twice in PBS and visualized via fluorescence microscopy or SIM as described previously ${ }^{36}$. In short, images were collected with a DeltaVision (GE Healthcare, Pittsburgh, USA) OMG Imaging System equipped with a Photometrics (Tucson, USA) Cascade II EMCCD camera (excitation: $405 \mathrm{~nm}$ and emission: $419-465 \mathrm{~nm}$ ).

Analysis of muropeptides obtained from sacculi. Sacculi were prepared from the K. stuttgartiensis cells $\left(0.81, \mathrm{OD}_{600} 1.1\right)$ enriched by density centrifugation as described above. Peptidoglycan was isolated largely as described before ${ }^{35}$. Briefly, cell pellets were boiled in $4 \%$ SDS for $60 \mathrm{~min}$ at $100^{\circ} \mathrm{C}$. After boiling for an additional $4 \mathrm{~h}$, sacculi were further stirred overnight at $37^{\circ} \mathrm{C}$ and then, SDS was washed out by ultracentrifugation. Sacculi were resuspended in $200 \mu \mathrm{l}$ of $50 \mathrm{mM}$ sodium phosphate buffer $\mathrm{pH} 4.5$ and digested overnight with $30 \mu \mathrm{g} \mathrm{ml}^{-1}$ muramidase (cellosyl, Hoechst) at $37^{\circ} \mathrm{C}$. Muramidase digestion was stopped by incubation in a boiling water bath $(5 \mathrm{~min})$. Coagulated protein was removed by centrifugation. The supernatants were mixed with $150 \mu \mathrm{l} 0.5 \mathrm{M}$ sodium borate $\mathrm{pH}$ 9.5 , and subjected to reduction of muramic acid residues into muramitol by sodium borohydride treatment $\left(10 \mathrm{mg} \mathrm{ml}^{-1}\right.$ final concentration, $30 \mathrm{~min}$ at room temperature). Samples were adjusted to $\mathrm{pH} 3.5$ with phosphoric acid. UPLC analyses of muropeptides were performed on an ACQUITY UPLC BEH C18 Column, $130 \mathrm{~A}$ 1.7, 2.1 and $150 \mathrm{~mm}$ (Waters, USA) and detected at $204 \mathrm{~nm}$. Muropeptides were separated using a linear gradient from buffer A (phosphate buffer $50 \mathrm{mM}, \mathrm{pH} 4.35$ ) to buffer B (phosphate buffer $50 \mathrm{mM}, \mathrm{pH} 4.95$ methanol $15 \%(\mathrm{v} / \mathrm{v})$ ) in $20 \mathrm{~min}$. Muropeptide purification was performed by HPLC on an Aeris peptide column $(250 \times 4.6 \mathrm{~mm} ; 3.6 \mu \mathrm{m}$ particle size; Phenomenex, USA). The identity of individual muropeptides was established by MALDI-TOF (Voyager DE-STR).

\section{References}

1. Vollmer, W., Blanot, D. \& De Pedro, M. Peptidoglycan structure and architecture. FEMS Microbiol. Rev. 32, 149-167 (2008).

2. Wagner, M. \& Horn, M. The Planctomycetes, Verrucomicrobia, Chlamydiae and sister phyla comprise a superphylum with biotechnological and medical relevance. Curr. Opin. Biotechnol. 17, 241-249 (2006).

3. König, E., Schlesner, H. \& Hirsch, P. Cell wall studies on budding bacteria of the Planctomyces/Pasteuria group and on a Prosthecomicrobium sp. Arch. Microbiol. 138, 200-205 (1984).

4. Liesack, W., König, H., Schlesner, H. \& Hirsch, P. Chemical composition of the peptidoglycan-free cell envelopes of budding bacteria of the Pirella/ Planctomyces group. Arch. Microbiol. 145, 361-366 (1986).

5. Cayrou, C., Raoult, D. \& Drancourt, M. Broad-spectrum antibiotic resisitance of Planctomycetes organisms determined by Etest. J. Antimicrob. Chemother. 65, 2119-2122 (2010).

6. McCoy, A. J. \& Maurelli, A. T. Building the invisible wall: updating the chlamydial peptidoglycan anomaly. Trends Microbiol. 14, 70-77 (2006).

7. Pilhofer, M. et al. Discovery of chlamydial peptidoglycan reveals bacteria with murein sacculi but without FtsZ. Nat. Commun. 4, 2856 (2013).

8. Liechti, G. W. et al. A new metabolic cell-wall labelling method reveals peptidoglycan in Chlamydia trachomatis. Nature 506, 507-510 (2014).

9. Jogler, C. et al. Planctomycetes comparative genomics: identification of proteins likely involved in morphogenesis, cell division and signal transduction. $J$. Bacteriol. 194, 6419-6430 (2012).

10. Guo, M. et al. Genomic evolution of 11 type strains within family Planctomycetaceae. PLoS ONE 9, e86752 (2014).

11. Lindsay, M. R. et al. Cell compartmentalization in planctomycetes: novel types of structural organization for the bacterial cell. Arch. Microbiol. 175, 413-429 (2001).

12. Sagulenko, E. et al. Structural studies of Planctomycete Gemmata obscuriglobus support cell compartmentalization in a bacterium. PLoS ONE 9, e91344 (2014).

13. Speth, D. R., van Teeseling, M. C. F. \& Jetten, M. S. M. Genomic analysis indicates the presence of an asymmetric bilayer outer membrane in Planctomycetes and Verrucomicrobia. Front. Microbiol. 3, 304 (2012). vested from the membrane bioreactor were concentrated and resuspended in $5 \mathrm{~m}$ $20 \mathrm{mM}$ HEPES buffer $\mathrm{pH} 7.8$ including $1.25 \mathrm{gl}^{-1}$ sodium bicarbonate. Afterwards, cell suspensions were made anoxic by applying under-pressure and flushing with $\mathrm{Ar} / \mathrm{CO}_{2}(95 \% / 5 \%)$ ten times. Then the cell suspensions were transferred to a $100 \mathrm{ml}$ fed-batch reactor inside an anaerobic chamber with an $\mathrm{Ar} / \mathrm{H}_{2}$ (95\%/5\%) atmosphere. $\mathrm{O}_{2}$ in the Ar in the anaerobic chamber was removed by passing Ar over a 
14. Devos, D. P. Re-interpretation of the evidence for the PVC cell plan supports a Gram-negative origin. Antonie Van Leeuwenhoek 105, 271-274 (2014).

15. Devos, D. P. PVC bacteria: variation of, but not exception to, the Gramnegative cell plan. Trends Microbiol. 22, 14-20 (2014).

16. Fuerst, J. A. The planctomycetes: emerging models for microbial ecology, evolution and cell biology. Microbiology. 141, 1493-1506 (1995).

17. Fuerst, J. A. \& Sagulenko, E. Keys to eukaryality: planctomycetes and ancestral evolution of cellular complexity. Front. Microbiol. 3, 167 (2012).

18. Forterre, P. \& Gribaldo, S. Bacteria with a eukaryotic touch: a glimpse of ancient evolution? Proc. Natl Acad. Sci. USA 107, 12739-12740 (2010).

19. Devos, D. P. \& Reynaud, E. G. Intermediate steps. Science 330, 1187-1188 (2010).

20. Santarella-Mellwig, R., Pruggnaller, S., Roos, N., Mattaj, I. W. \& Devos, D. P. Three-dimensional reconstruction of bacteria with a complex endomembrane system. PLoS Biol. 11, e1001565 (2013).

21. Kartal, B. et al. Molecular mechanisms of anaerobic ammonium oxidation. Nature 479, 127-130 (2011).

22. Francis, C. A., Beman, J. M. \& Kuypers, M. M. M. New processes and players in the nitrogen cycle: the microbial ecology of anaerobic and archeal ammonia oxidation. ISME J. 1, 19-27 (2007).

23. Kartal, B., Kuenen, J. G. \& van Loosdrecht, M. C. M. Sewage treatment with anammox. Science 328, 702-703 (2010).

24. van Niftrik, L. et al. Combined structural and chemical analysis of the anammoxosome: a membrane-bounded intracytoplasmic compartment in anammox bacteria. J. Struct. Biol. 161, 401-410 (2008).

25. Neumann, S. et al. Isolation and characterization of a prokaryotic cell organelle from the anammox bacterium Kuenenia stuttgartiensis. Mol. Microbiol. 94, 794-802 (2014).

26. van Teeseling, M. C. F. et al. A new addition to the cell plan of anammox bacteria: "Candidatus Kuenenia stuttgartiensis" has a protein surface layer as the outermost layer of the cell. J. Bacteriol. 196, 80-89 (2014).

27. van Niftrik, L. et al. Cell division ring, a new cell division protein and vertical inheritance of a bacterial organelle in anammox planctomycetes. $\mathrm{Mol}$. Microbiol. 73, 1009-1019 (2009).

28. Neumann, S., van Teeseling, M. C. F. \& van Niftrik, L. in Planctomycetes: Cell Structure, Origins and Biology. (ed Fuerst, J. A.) 89-123 (Humana Press/ Springer, 2013).

29. Lombard, V., Golaconda Ramula, H., Drula, E., Coutinho, P. M. \& Henrissat, B. The carbohydrate-active enzymes database (CAZy) in 2013. Nucleic Acids Res. 42, D490-D495 (2014).

30. Hu, Z., van Alen, T., Jetten, M. S. M. \& Kartal, B. Lysozyme and Penicillin inhibit the growth of anaerobic ammonium-oxidizing Planctomycetes. Appl. Environ. Microbiol. 79, 7763-7769 (2013).

31. Al-Amoudi, A. et al. Cryo-electron microscopy of vitreous sections. EMBO J. 23, 3583-3588 (2004).

32. Johnson, L. N. \& Phillips, D. C. Structure of some crystalline lysozymeinhibitor complexes determined by X-ray analysis at 6 Angstrom resolution. Nature 206, 761-763 (1965).

33. De Pedro, M. A., Quintela, J. C., Höltje, J.-V. \& Schwarz, H. Murein segregation in Escherichia coli. J. Bacteriol. 179, 2823-2834 (1997).

34. Strous, M. et al. Deciphering the evolution and metabolism of an anammox bacterium from a community genome. Nature 440, 790-794 (2006).

35. Cava, F., de Pedro, M. A., Lam, H., Davis, B. M. \& Waldor, M. K. Distinct pathways for modification of the bacterial cell wall by non-canonical D-amino acids. EMBO J. 30, 3442-3453 (2011).

36. Kuru, E. et al. In situ probing of newly synthesized peptidoglycan in live bacteria with fluorescent D-amino acids. Angew. Chem. Int. Ed. Engl. 51, 12519-12523 (2012).

37. Siegrist, M. S. et al. D-amino acid chemical reporters reveal peptidoglycan dynamics of an intracellular pathogen. ACS Chem. Biol. 8, 500-505 (2013).
38. Gordon, E. et al. Crystal structure of UDP-N-acetylmuramoyl-L-alanyl-Dglutamate:meso-Diaminopimelate ligase from Escherichia coli. J. Biol. Chem. 276, 10999-11006 (2000).

39. Jeske, O. et al. Planctomycetes do possess a peptidoglycan cell wall. Nat. Commun. 6: 7116 doi: 10.1038/ncomms8116 (2015).

40. Kartal, B., Geerts, W. \& Jetten, M. S. M. in Methods in Enzymology Vol. 486 (ed Klotz, M. G.) 89-109 (Academic, 2011).

41. van de Graaf, A. A., de Bruijn, P., Robertson, L. A., Jetten, M. S. M. \& Kuenen, J. G. Autotrophic growth of anaerobic ammonium-oxidizing micro-organisms in a fluidized bed reactor. Microbiology (UK) 142, 2187-2196 (1996).

42. Schmid, M. et al. Molecular evidence for genus level diversity of bacteria capable of catalyzing anaerobic ammonium oxidation. Syst. Appl. Microbiol. 23, 93-106 (2000).

43. Neef, A., Amann, R. I., Schlesner, H. \& Schleifer, K. H. Monitoring a widespread bacterial group: in situ detection of planctomycetes with $16 \mathrm{~S}$ rRNA-targeted probes. Microbiology. 144, 3257-3266 (1998).

\section{Acknowledgements}

Miguel A. de Pedro, Karin Aistleitner, Matthias Horn, David Kysela, Yoan Diekmann and José Pereira Leal are acknowledged for discussions. We thank Joachim Reimann and Christina Ferousi for the Kuenenia stuttgartiensis cells and Thomas Kieselbach for mass spectrometry analysis. We thank the Indiana University Light Microscopy Imaging Center for their help with OMX super-resolution microscopy, supported by National Institutes of Health grant S10RR028697. Katharina Ettwig, Huub Op den Camp, Joachim Reimann and Daan Speth are acknowledged for critical reading of the manuscript. M.C.F.v.T. is supported by ERC grant 232937 and R.J.M. by the 2012 Spinoza Grant both awarded to Mike Jetten. E.K. and Y.V.B. are supported by NIH grant GM051986. F.C. and A.E. are supported by MIMS, KAW, Kempe Foundation and the Swedish Research Council. B.K. and L.v.N. are supported by NWO VENI grant 863.11.003 and 863.09.009, respectively.

\section{Author contributions}

M.C.F.v.T., R.J.M., B.K. and L.v.N. designed the project; R.J.M. performed the cryoTEM experiments. M.C.F.v.T. isolated sacculi and performed the lysozyme experiment; M.C.F.v.T. and B.K. set-up fed-batch incubations with D-amino acid probes; E.K., Y.V.B. and M.S.V. provided the D-amino acid probes; E.K. performed click chemistry and visualization of the incorporation experiments (fluorescence microscopy and SIM); M.C.F.v.T., R.J.M. and A.E. prepared sacculi for muropeptide analysis; A.E. performed UPLC and MS analysis; A.E. and F.C. analysed the muropeptides; M.C.F.v.T., R.J.M., E.K., A.E., F.C., Y.V.B., M.S.V., B.K. and L.v.N. analysed data; M.C.F.v.T., R.J.M., B.K. and L.v.N. wrote the paper with input from all authors.

\section{Additional information}

Supplementary Information accompanies this paper at http://www.nature.com/ naturecommunications

Competing financial interests: The authors declare no competing financial interests.

Reprints and permission information is available at http://npg.nature.com/ reprintsandpermissions/

How to cite this article: van Teeseling, M. C. F. et al. Anammox Planctomycetes have a peptidoglycan cell wall. Nat. Commun. 6:6878 doi: 10.1038/ncomms7878 (2015)

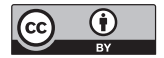

This work is licensed under a Creative Commons Attribution 4.0 International License. The images or other third party material in this article are included in the article's Creative Commons license, unless indicated otherwise in the credit line; if the material is not included under the Creative Commons license, users will need to obtain permission from the license holder to reproduce the material. To view a copy of this license, visit http://creativecommons.org/licenses/by/4.0/ 